\title{
Aging and interferon gamma response drive the phenotype of neutrophils in the inflamed joint
}

Ricardo Grieshaber-Bouyer* (1, 2, 3, 4), Tarik Exner (1, 2), Nicolaj S. Hackert (1, 2), Felix A. Radtke (1, 2, 4), Scott A. Jelinsky (5), Olha Halyabar (6), Alexandra Wactor (4), Elham Karimizadeh (4), Jorge Schettini (5), A. Helena Jonsson (4), Deepak A. Rao (4), Lauren A. Henderson (6), Carsten Müller-Tidow (3, 7), Hanns-Martin Lorenz (1), Guido Wabnitz (2), James A. Lederer (8), Angela Hadjipanayis (5) and Peter A. Nigrovic* $(4,6)$

(1) Division of Rheumatology, Department of Medicine V, Hematology, Oncology and Rheumatology, Heidelberg University Hospital, Heidelberg, Germany

(2) Institute of Immunology, Heidelberg University Hospital, Heidelberg, Germany

(3) Molecular Medicine Partnership Unit, European Molecular Biology Laboratory (EMBL), University of Heidelberg, Heidelberg, Germany

(4) Division of Rheumatology, Inflammation, and Immunity, Brigham and Women's Hospital, Harvard Medical School, Boston MA, USA

(5) Pfizer, Inc., Cambridge, MA, USA

(6) Division of Immunology, Boston Children's Hospital, Boston MA, USA

(7) Department of Medicine V, Hematology, Oncology and Rheumatology, Heidelberg University Hospital, Heidelberg, Germany

(8) Department of Surgery, Brigham and Women's Hospital, Harvard Medical School, Boston MA, USA

* Correspondence to R.G-B and P.A.N.:

Ricardo Grieshaber-Bouyer, MD

Division of Rheumatology

Department of Medicine V (Hematology, Oncology and Rheumatology)

Heidelberg University Hospital

Im Neuenheimer Feld 410

69120 Heidelberg, Germany

Email: ricardo.grieshaberbouyer@med.uni-heidelberg.de

Peter A. Nigrovic, MD

Chief, Division of Immunology, Boston Children's Hospital

Karp Family Research Building, Room 10211

One Blackfan Circle

Boston, MA 02115, USA

Email: peter.nigrovic@childrens.harvard.edu 


\begin{abstract}
Objectives: Neutrophils are typically the most abundant leukocyte in arthritic synovial fluid. We sought to understand changes that occur in neutrophils as they migrate from blood to joint.

Methods: We performed RNA sequencing of neutrophils from healthy human blood, arthritic blood, and arthritic synovial fluid, comparing transcriptional signatures with those from murine $\mathrm{K} / \mathrm{BxN}$ serum transfer arthritis. We employed mass cytometry to quantify protein expression and sought to reproduce the synovial fluid phenotype ex vivo in cultured healthy blood neutrophils.

Results: Blood neutrophils from healthy donors and patients with active arthritis exhibited largely similar transcriptional signatures. By contrast, synovial fluid neutrophils exhibited more than 1,600 differentially expressed genes. Gene signatures identified a prominent response to interferon gamma (IFNy), as well as to tumor necrosis factor, interleukin 6, and hypoxia, in both humans and mice. Mass cytometry also found healthy and arthritic donor blood neutrophils largely indistinguishable but revealed a range of neutrophil phenotypes in synovial fluid defined by downregulation of CXCR1 and upregulation of FcYRI, HLA-DR, PD-L1, ICAM-1 and CXCR4. Reproduction of key elements of this signature in cultured blood neutrophils required both IFNY and prolonged culture.
\end{abstract}

Conclusions: Circulating neutrophils from arthritis patients resemble those from healthy controls, but joint fluid cells exhibit a network of changes, conserved across species, that implicate IFNY response and aging as complementary drivers of the synovial neutrophil phenotype.

\title{
Keywords
}

Inflammatory arthritis, synovial fluid, neutrophils

\section{KEY MESSAGES}

\section{What is already known about this subject?}

- Neutrophils are central in the effector phase of inflammatory arthritis but their phenotypic heterogeneity in inflamed synovial fluid is poorly understood.

\section{What does this study add?}

- $\quad$ RNA-seq and mass cytometry identify a hallmark phenotype of neutrophils in synovial fluid consisting of upregulated ICAM-1, HLA-DR, PD-L1, Fc receptors and CXCR4.

- Transcriptomics highlight an IFNy response signature conserved across humans and mice.

- In vitro experiments implicate aging and IFNy as complementary factors orchestrating the synovial fluid neutrophil phenotype.

\section{How might this impact on clinical practice or future developments?}

- Understanding the specific features of neutrophils in the arthritic joint may disclose opportunities for safe therapeutic targeting of this lineage. 


\section{INTRODUCTION}

Inflammatory arthritis encompasses a broad spectrum of diseases affecting adults and children[1]. The pathogenesis of non-infectious arthritis is correspondingly varied, with upstream mechanisms that include autoantibodies, T cells, autoinflammatory mechanisms, and crystals[2]. Despite this remarkable pathogenic diversity, a ubiquitous feature of arthritic joint fluid is an abundance of neutrophils, a canonical innate immune effector cell required for immune defense but also for many forms of pathogenic inflammation.

Compelling evidence confirms that neutrophils are key pathogenic contributors in arthritis. Neutrophils from human joints exhibit altered surface markers and function consistent with activation[3-7]. Synovial neutrophils elaborate pro-inflammatory factors such as interleukin (IL)-1, leukotriene B4, citrullinated peptides, and neutrophil extracellular traps[8-11]. Neutrophils activated by adherent immune complexes degrade articular cartilage[12]. Finally, mice with defects specific to the neutrophil compartment - for example, depleted of neutrophils, congenitally deficient in neutrophils, with neutrophils lacking key effector molecules, or subject to neutrophil migratory blockade - exhibit dense resistance to experimental arthritis[11, 13-17]. Therefore, understanding the phenotype of synovial fluid neutrophils is essential to understanding the biology of arthritis and may reveal novel opportunities for therapeutic intervention[18].

Advances in cellular characterization offer new ways to understand neutrophils. Transcriptomic analysis provides a hypothesis-independent examination of the activity of cells at the gene expression level, informing the relationships between populations of cells. For example, studies using single-cell RNA sequencing (scRNAseq) recently established that murine neutrophils represent a single lineage, differentiating along a developmental continuum termed 'neutrotime', rather than a branched network of committed subtypes[19]. Indeed, aging is well recognized as a modulator of neutrophil phenotype and function[20, 21]. Importantly, however, the relationship between a neutrophil's transcriptome and its surface protein signature varies markedly with context[19]. For example, neutrophil activation is accompanied by rapid mobilization of the surface integrin CD11b from an intracellular pool and cleavage-mediated loss of the surface selectin CD62L[22]. Mass cytometry (cytometry by time of flight, CyTOF) allows simultaneous determination of dozens of surface and intracellular markers in each cell, albeit restricted by investigator choice as to the markers most likely to prove informative[23].

To understand the changes that occur in neutrophils as they enter the inflamed joint, we applied low-input RNAseq to purified neutrophils, sorting cells by a known dichotomous surface marker of undetermined function, CD177, to eliminate potential confounding by variation in the CD177 ${ }^{\text {pos }}$ neutrophil fraction within the population[24]. We compared human neutrophils with scRNAseq transcriptome data from murine neutrophils, both circulating and from autoantibody-mediated neutrophil-driven K/BxN serum transfer arthritis[25]. We employed CyTOF to define markers of neutrophil differentiation and function, followed by confirmatory in vitro studies using flow cytometry. We establish that blood neutrophils from healthy and arthritic donors are largely similar but find that synovial fluid neutrophils differ markedly from blood neutrophils in a manner that implicates both interferon gamma (IFNY) response and cell aging in the resulting phenotype. 


\section{METHODS}

Demographic characteristics and materials and methods are available in the supplementary materials.

\section{RESULTS}

\section{Transcriptomic characterization of circulating neutrophils}

We performed low-input RNAseq on blood neutrophils from 15 healthy and 16 arthritic donors (Supplementary Table 1), calculating Pearson correlation coefficients between samples based on the expression of all genes. Hierarchical clustering of these correlation coefficients revealed no separation by disease state, suggesting little divergence of transcriptional phenotype (Figure 1A). At an FDR of 0.05 (indicated by red line), blood neutrophils from healthy and arthritic donors differed in only three genes: DNAJB9 (DnaJ Heat Shock Protein Family (Hsp40) Member B9), DDIT4 (DNA damage inducible transcript 4) and SCO2 (Synthesis of Cytochrome C Oxidase 2), all modestly upregulated in arthritis (Figure 1B, C).

\section{Synovial fluid neutrophils display an IFNY response}

We performed the same analysis in 16 paired contemporaneous peripheral blood and synovial fluid samples from patients with active arthritis requiring therapeutic joint aspiration. Hierarchical clustering revealed separation into two groups as a function of location (Figure 2A). At $\mid \log$ fold change $\mid \geq 1$ and FDR $0.05,1,657$ genes were differentially expressed, of which 939 were downregulated and 718 upregulated (Figure 2B).

To understand these genes in terms of functional programs, we employed Gene Set Enrichment Analysis using the established 50 hallmark gene sets. Signatures of TNF and IL-6 response were easily detected, as was response to hypoxia (Figure 2C). However, the most prominent gene set in synovial fluid neutrophils was IFNy response (Figure 2C, 2D). Analysis of genes up-regulated in response to IFNY (HALLMARK_INTERFERON_GAMMA_RESPONSE) revealed that most (93/175) expressed IFNy target genes were highly induced in synovial fluid neutrophils, including the class II molecules CD74, HLA-DMA, HLA-DRB1 and HLA-DQA1; CD274 (encoding PD-L1); and FCGR1A (encoding CD64, the high-affinity lgG receptor FcyRI) (Figure 2E). These observations show that phenotypic deviation of neutrophils in arthritis is primarily in the joints rather than in the circulation, at least at the transcriptional level, and suggest a prominent role for IFNy in driving the phenotype of synovial fluid neutrophils.

\section{Conserved responses of human and murine neutrophils in inflammatory arthritis}

Neutrophils are indispensable for onset and perpetuation of joint inflammation in mice[8-11, 1416]. To test whether the transcriptional changes we observed in human synovial neutrophils are conserved across species, we compared our human dataset to a microarray-based transcriptional atlas of neutrophils from the blood of healthy mice and joints of mice undergoing $\mathrm{K} / \mathrm{BxN}$ serum transfer arthritis[25]. We restricted the combined dataset to 5,520 one-to-one gene orthologs according to ENSEMBL version 100[26]. Of genes with orthologs significantly upregulated in human (578) and murine (226) synovial fluid neutrophils, 97 were shared across species, far more than expected by chance (95\% confidence interval for chance overlap 16-34 genes as defined 
by random resampling 20,000 times, $P=2.7 \times 10^{-7}$, Figure 3A). Similarly, downregulated genes across human (774) and mouse (174) neutrophils in synovial fluid shared significant overlap with 75 genes, compared to $23-41$ expected by chance $\left(P=1.3 \times 10^{-11}\right.$, Figure 3A). In murine synovial fluid neutrophils, enhanced expression was observed in IFNy target genes including CD274 (encoding PD-L1) and the MHC class II gene HLADQB1; indeed an IFNy signature was one of the key functional patterns observed, with highly skewed representation of IFNY response genes (adjusted $P<0.001$, Figure 3B,C). These findings establish that gene expression changes, including an IFNy response signature, are shared by human and mice synovial fluid neutrophils.

\section{CyTOF confirms joint-specific activation of human neutrophils in inflammatory arthritis}

We created a custom CyTOF panel containing 39 human surface and intracellular markers related to neutrophil activation, chemokine receptors, antigen presentation, adhesion factors and costimulatory molecules (Supplementary Methods). CyTOF studies were performed in 33 samples (9 healthy volunteer donors, 8 blood samples from patients with inflammatory arthritis, and 16 synovial fluid samples, including 7 contemporaneous blood/synovial fluid pairs; Supplementary Table 1).

To analyze global data structure, we extracted median expression values for each protein in each sample and calculated Spearman correlation coefficients between samples based on expression data. Hierarchical clustering revealed complete overlap in peripheral blood neutrophils between healthy donors and patients with inflammatory arthritis, indicating few systematic differences in global protein expression (Figure 4A). Correspondingly, we found differential expression only of a single marker, CD64, between healthy and arthritic donor peripheral blood neutrophils after correction for multiple comparisons (Figure 4C). These results mirror our transcriptomic findings and show similarity of blood neutrophils between healthy and arthritic donors.

By contrast, comparison of blood and synovial fluid revealed a strong separation driven by tissue (Figure 4B). This separation was driven by multiple differentially expressed proteins in synovial fluid neutrophils including downregulation of CXCR1 and upregulation of the integrin CD11C, PDL1, ICAM-1, HLA-DR, the low-affinity Fc receptor CD32 (FcyRII) and CXCR4 (CD184), the receptor for CXCL12/SDF-1 that retains neutrophils in inflamed sites [27] (Figure 4D,E). CD64 was also overexpressed but did not reach significance compared to arthritic donor blood due to already higher expression (Figure 4E). Compared to healthy blood neutrophils, synovial fluid neutrophils overexpressed CD64, the activation and lineage markers CD66b and CD15, the LPS co-receptor CD14, and the integrin CD49d (Figure 4E).

PD-L1, HLA-DR and CD64 are upregulated in neutrophils exposed to IFNy, consistent with our transcriptomic signature data[28-34]. The IL-8 receptor CXCR1 was downregulated, potentially reflecting agonist-mediated internalization of this G-protein-coupled receptor. No change was noted in granule proteins, including for primary (azurophilic) granules (including MPO, proteinase 3 [PR3], arginase 1), secondary granules (including LL-37 / cathelicidin, CD177, OLFM4), and tertiary granules (arginase 1). Results for all markers are shown in Supplementary Figure 3.

We investigated how well expression differences in RNA and protein match each other. We found that downregulation of $C X C R 1$ and upregulation of CXCR4, ICAM1, HLA-DRA, HLA-DRB1, HLA$D R B 5$ and CD274 (encoding PD-L1) were highly concordant between RNA and protein (Figure 
4F). Upregulation of FCGR1A (CD64) and FCGR2B (CD32) was also observed on both RNA and protein level but was significant only at either gene (FCGR1A) or protein (CD32) level. This set of genes and their protein products thus constitute hallmarks of the synovial fluid neutrophil phenotype.

\section{Continuous and discrete neutrophil phenotypes}

To define neutrophil heterogeneity at the single-cell level, we performed Uniform Manifold Approximation and Projection (UMAP) dimensionality reduction on our CyTOF data. Initial results were dominated by the two known dichotomously expressed neutrophil proteins, CD177 and OLFM4 (Supplementary Figure 4A). Cells from peripheral blood and synovial fluid were evenly distributed across CD177 $7^{\text {pos/neg }}$ and OLFM $4^{\text {pos/neg }}$ populations in the UMAP embedding, suggesting that the phenotypic changes distinguishing blood and synovial fluid neutrophils operate evenly across these markers (Supplementary Figure 4B). Accordingly, we did not detect any significant differences in frequency of neutrophil subsets defined by CD177 or OLFM4 between blood and synovial fluid (Supplementary Figure 4C).

To neutralize this dominant impact, we excluded CD177, OLFM4, and the CD177-anchored enzyme PR3 from consideration and repeated dimensionality reduction. We observed a striking separation between resting blood neutrophils and synovial fluid cells, single-cell findings that mirrored our transcriptomic results (Figure 5A). Synovial fluid neutrophils concentrated in two primary clusters, termed here SFN1 and SFN2 and observed across individual donors (Figure 5A and Supplemental Figure 5). For analysis purposes, we forced neutrophils into $k=20$ clusters, again excluding CD177, OLFM4, and PR3, with the goal of maximizing the opportunity to identify distinct phenotypic states (Figure 5B). Individual markers varied among the 20 clusters, confirming neutrophil heterogeneity at the single-cell level (Figure 5C). Examining the frequency of cells belonging to each cluster, we observed considerable divergence among donors, limiting statistical power in this relatively small sample size. Clusters 10-12 were particularly overrepresented in synovial fluid, representing the bulk of neutrophils in SFN2 (Figure 5D). Neutrophils in Clusters 10-11 expressed high levels of CXCR4 (CD184) and Cluster 12 cells additionally expressed the IFNy markers HLA-DR, PD-L1 and CD64. The SFN1 population was contained within Cluster 2 and was driven primarily by a single donor, although it trended higher across multiple samples in synovial fluid vs. blood. By contrast, blood neutrophils were enriched for Clusters 1, 8, 9, and 16, with only Cluster 9 (expressing high levels of granule proteins) and Cluster 16 (expressing granule proteins, OLFM4, and CD124, the alpha chain of the IL-4 and IL13 receptors) achieving statistical significance (Figure 5D).

Expression of differentially expressed markers between blood and synovial fluid revealed that most markers follow expression gradients (Figure 5E). Broadly, two gradients could be observed: a gradient from top to bottom that included many granule proteins and likely reflecting maturation (CD10, Nrf2, arginase 1, CD11a, elastase, LL-37, CD31, MPO, OLFM4, CD177, CD184/CXCR4) and a gradient from left to right likely reflecting activation (CD66b, CD11b, CD15, CD16, CXCR1, CD45) (Supplementary Figure 5). Small populations of interest included Clusters 8 and 19 (expressing TCR $\alpha \beta$, equally rare in blood and synovial fluid) and Cluster 6, expressing VEGFR1 and therefore potentially representing pro-angiogenic neutrophils[35], significantly increased in synovial fluid compared to healthy blood. 
Notably, not all upregulated markers were expressed on the same cells. For example, CXCR4high neutrophils expressed variable amounts of HLA-DR and PD-L1 (Figure 5E). Correlating expression intensity on a per-sample bulk level revealed a positive correlation between markers defining the core synovial fluid phenotype: HLA-DR, ICAM-1, CXCR4 and CD32 (Figure 5F). Analysis at the single-cell level confirmed a correlation between general activation markers CD11b, CD15 and CD66b and within a cluster of granule proteins (MPO, LL-37, Nrf2, Elastase) but not between CXCR4, HLA-DR and PD-L1 (Figure 5G).

Together, these results show that CXCR4+, HLA-DR+ and PD-L1+ neutrophils are expanded in inflamed synovial fluid and that expression of these markers peaks in different cells, confirming that the inflamed environment features divergent neutrophil phenotypes.

\section{IFNy and aging drive blood neutrophils toward a synovial fluid phenotype}

Since both transcriptomic and proteomic analysis revealed a strong IFNy response signature in synovial fluid neutrophils, we hypothesized that stimulation with IFNy could recapitulate the synovial fluid phenotype in healthy blood neutrophils. As expected, viability dropped from nearly $100 \%$ at beginning of culture to $71 \%$ after 2 days of culture at $37^{\circ} \mathrm{C}$. Stimulation with IFNy significantly extended neutrophil survival to $87 \%$ (Figure 6A).

IFNy stimulation prevented downregulation of CD32 and significantly upregulated CD64, ICAM1, HLA-DR and PD-L1 (Figure 6B). CXCR4 expression was not detectable in freshly isolated neutrophils but increased with time in culture, consistent with its known role as a marker of neutrophil aging[21]. Interestingly, CXCR4 expression was reduced by cytokine stimulation, indicating either an impact on CXCR4 expression specifically or a broader effect on the neutrophil aging program (Figure 6B).

Based on those findings, we hypothesized that cytokine stimulation and aging were complementary in establishing the synovial fluid neutrophil phenotype. We therefore analyzed unstimulated and stimulated neutrophils together in a single diffusion map. This analysis revealed a marked divergence in phenotypes between cells left unstimulated and those incubated with IFNy (Figure 6C). CXCR4 expression was highest at the most distant pole of the unstimulated trajectory (Figure 6D). Conversely, IFNy robustly upregulated HLA-DR, PD-L1 and ICAM-1 (Figure 6D). Thus, the combination of aging and exposure to IFNy, but not either alone, yielded a neutrophil phenotype resembling that of synovial fluid neutrophils.

\section{DISCUSSION}

Synovial fluid neutrophils are the hallmark of inflammatory arthritis[36]. We employed low-input RNA sequencing and CyTOF to characterize neutrophils from healthy donor blood and from blood and synovial fluid of patients with active arthritis. Whereas circulating neutrophils exhibited few changes with disease state, synovial fluid neutrophils displayed consistent phenotypic deviation implicating two conceptually orthogonal influences: response to local mediators, most prominently IFNy, and cell aging.

The marked alteration in mRNA expressed by synovial fluid neutrophils is consistent with the growing understanding of neutrophils as highly dynamic cells that remain transcriptionally active throughout their lifespan[19, 37, 38]. This adaptability may be of particular consequence in 
neutrophils recruited to inflamed sites such as the arthritic joint, since cytokines can prolong neutrophil half-life from a baseline of 8-20 hours to several days[39].

Transcriptional signatures observed here included response to mediators of established importance in arthritis, including TNF and IL-6, as well as to hypoxia, a known feature of the synovial environment[40]. The role of IFNy in arthritis is less well understood. Studies performed more than 20 years ago implicated IFNy in expression of the high-affinity Fc receptor FcyRI (CD64) on synovial fluids neutrophils[6]. Potential IFNy sources in arthritis suggested by human and/or murine studies include CD4 T cells (IFNy is the hallmark Th1 cytokine), CD8 T cells, NK cells, and NKT cells[41-46]. Experimental overexpression of IFNy in the joint accelerates cartilage injury through upregulation of IgG Fc receptors and therefore enhanced susceptibility to immune complex injury[47]. However, IFNy-deficient mice exhibit normal susceptibility to IgG-mediated $\mathrm{K} / \mathrm{BxN}$ serum transfer arthritis, while IFNy blockade or IFNy receptor deficiency accelerates the onset and severity of collagen-induced arthritis[44, 48, 49]. Trials of recombinant IFNy in rheumatoid arthritis found at best modest disease amelioration[50, 51]. These findings reflect the net impact of IFNy on multiple lineages and remain compatible with the possibility that neutrophil exposure to IFNy in arthritis is pro-inflammatory (for example, through upregulation of surface Fc receptors and HLA-DR), anti-inflammatory (for example, through up-regulation of the $\mathrm{T}$ cell inhibitor PD-L1), or both.

Comparing the transcriptional signature of human and murine neutrophils, we observed substantial overlap, including shared presence of an IFNy signature in synovial fluid neutrophils, supporting the human relevance of extensive murine work defining the role of neutrophils in arthritis[11, 13-16]. This conclusion is important because performance of unambiguous human studies is complicated by the lack of neutrophil-specific therapeutics. Cross-species similarity is further echoed in murine neutrophil scRNAseq data, where differences between healthy and arthritic blood neutrophils were small whereas differences between arthritic blood and synovial neutrophils were large[19]. Whereas the neutrotime signature cannot be extrapolated directly to bulk RNAseq data, downregulation of early-neutrotime transcripts such as LCN2, CAMP, and $C D 177$ further supports the suggestion that human synovial fluid neutrophils - like their murine counterparts - skew similarly skew toward an aged phenotype reflecting prolonged survival in the inflamed joint environment[19].

Of particular interest is the marked cell-to-cell heterogeneity revealed by CyTOF. The clusters reported here reflect investigator-chosen analytical parameters and therefore are best regarded as one snapshot of this complex population rather than as discrete subsets. The data show that neutrophils within the inflamed joint differ phenotypically from each other as well as from those in blood. Dimensionality reduction by UMAP identified 2 broad populations: SFN1, resembling circulating neutrophils, and SFN2, a more abundant group often bearing markers associated with the IFNy signature. We speculate that these populations reflect a chronological progression, with SFN1 representing recent arrivals that evolve in SFN2 cells with exposure to the inflamed synovial environment and time. This suggestion is consistent with greater expression within SFN2 of the maturity marker CD10 and the aging marker CXCR4, although SFN2 neutrophils remain internally diverse[52].

We applied both inflammatory stimuli and time to cultured healthy donor blood neutrophils. Indeed, two orthogonal signals were noted: IFNy exposure upregulated hallmark SFN2 proteins 
such as HLA-DR, PD-L1, and CD64, while aging was required to yield the second key SFN2 marker, CXCR4 (interestingly partially suppressed by IFNY). Further study will be required to confirm the parallels between these findings and the arthritis context, but the data nevertheless support the conceptual model that the neutrophil phenotypes observed in human synovial fluid represent an integration of inflammatory stimuli and aging in cells recruited in an ongoing manner to the inflamed joint.

Our work has several limitations. RNAseq studies employed bulk sorted neutrophils, enabling us to identify transcripts in depth but prohibiting us from calculating developmental trajectories. Future studies using scRNAseq will be important to define the ontological relationships among joint fluid neutrophils. CyTOF studies employed a set of antigens reflecting investigator choice; for technical reasons, not all antigens proved interpretable, and it is likely that many informative antigens were omitted. Our data do not detail epigenetic reprograming of neutrophils, and we did not characterize the function of the heterogeneous groups identified in arthritic synovial fluid. Despite these limitations, the results represent a uniquely granular examination of the transcriptional and surface/intracellular phenotype of human arthritic neutrophils, setting the stage for the next set of phenotypic and functional studies toward the ultimate goal of identifying targetable pathways for therapeutic neutrophil blockade in arthritis.

\section{Acknowledgements}

We thank Adam T. Chicoine and the Brigham and Women's Hospital cell sorting facility for cell sorting and Jüri Habicht for technical assistance.

\section{Funding}

This work was supported by funds from the state of Baden-Wuerttemberg within the Centers for Personalized Medicine Baden-Wuerttemberg (ZPM), an MD fellowship from Boehringer Ingelheim Fonds, a physician-scientist development grant from the Medical Faculty Heidelberg, and a research grant from the German Society for Rheumatology (DGRh), and a Gilead grant (to R.G-B.). T.E. was funded by the MD/PhD Programme of Heidelberg Faculty of Medicine. F.A.R. was supported by an MD fellowship from Boehringer Ingelheim Fonds. P.A.N is supported by NIH/NIAMS awards 2R01AR065538, R01AR075906, R01AR073201, R21AR076630, 2P30AR070253, R56AR065538, NIH/NHLBI R21HL150575, the Fundación Bechara, the Samara Jan Turkel Center for Pediatric Autoimmune Diseases at Boston Children's Hospital, and the Arbuckle Family Fund for Arthritis Research. Some data reported were collected with the support of an investigator-initiated research grant to P.A.N. from Pfizer, Inc., but final decision about data analysis and publication remained with P.A.N.

\section{Competing Interests}

R.G.-B. received research support from Gilead. S.A.J. and A.H. are employees of Pfizer, Inc. H.M.L. received research grants from Abbvie, Pfizer, Novartis, Sobi, Roche/Chugai, Gilead, Galapagos, GSK, UCB, MSD, and BMS. P.A.N. has received investigator-initiated research grants from AbbVie, BMS, Novartis, Pfizer and Sobi; consulting fees from BMS, Cerecor, Miach Orthopedics, Novartis, Pfizer, Quench Bio, Sigilon, Simcere, Sobi, XBiotech, and Exo 
Therapeutics; royalties from UpToDate Inc. and the American Academy of Pediatrics; and salary support from the Childhood Arthritis and Rheumatology Research Alliance.

\section{Patient consent for publication}

Not required.

\section{Ethics approval}

EDTA-anticoagulated blood from healthy donors was collected under IRB-approved protocols (BWH-2008P000427 and Heidelberg S-285/2015). Simultaneous blood and synovial fluid samples were obtained with written consent from patients with inflammatory arthritis who underwent diagnostic and/or therapeutic joint aspiration (BCH-P00005723 and BWH2006P001068). Patients or the public were not involved in the design, or conduct, or reporting, or dissemination plans of our research.

\section{Data availability statement}

All relevant data is included in the article and online supplemental information. RNA-sequencing data will be made publicly available upon publication.

\section{FIGURE LEGENDS}

Figure 1. Transcriptomic similarity of blood neutrophils from healthy controls and patients with inflammatory arthritis

(A) Hierarchical clustering of Pearson correlation coefficients between individual blood samples based on the expression of all genes reveals complete overlap between the two groups. (B) Volcano plot of differentially expressed genes (at FDR 0.05) between blood neutrophils from healthy and arthritic donors. (C) DNAJB9, DDIT4 and SCO2 are overexpressed in blood of inflammatory arthritis patients compared to healthy controls. $N=15$ healthy controls, $N=16$ arthritis patients.

\section{Figure 2. Synovial fluid neutrophils are enriched for IFNy response genes}

(A) Hierarchical clustering of Pearson correlation coefficients between paired peripheral blood and synovial fluid samples based on the expression of all genes shows strong separation based on tissue. (B) 1,657/6,350 genes are differentially expressed at $\log _{2}$ fold change $\geq 1$ and FDR of 0.05 between peripheral blood and synovial fluid neutrophils. (C) Gene Set Enrichment Analysis of differentially expressed genes in synovial fluid vs. blood neutrophils. (D) Enrichment plot of the IFNy response signature in synovial fluid neutrophils. (E) Expression heatmap of IFNy response genes in synovial fluid neutrophils reveals strong separation between blood and synovial fluid. $\mathrm{N}$ $=16$ paired blood and synovial fluid inflammatory arthritis samples.

\section{Figure 3. Cross species analysis of neutrophil gene expression in inflamed synovial fluid}

(A) Depicted is the $\log _{2}$ fold change of gene expression in human ( $x$ ) vs. murine (y) synovial fluid neutrophils compared to blood neutrophils. Only genes with with one-to-one orthologs are shown and genes with adjusted $P<0.05$ in both comparisons and $\mid \log _{2}$ fold change $\mid \geq 0.75$ are 
highlighted. Genes are conservatively colored by highest P value. (B) Significantly differentially expressed genes were ranked by $\log _{2}$ fold change and Gene Set Enrichment Analysis was performed on hallmark gene sets. (C) Enrichment plot of the hallmark gene set "Interferon gamma response". Only genes with one-to-one orthologs between mice and humans are shown; for gene symbols, the human symbol is shown.

\section{Figure 4. Mass cytometry analysis of neutrophils}

(A) Hierarchical clustering of Spearman correlation coefficients between blood neutrophils from healthy donors and patients with inflammatory arthritis based on global neutrophil protein expression. (B) Differential expression analysis of global neutrophil marker expression in the peripheral blood. (C) Hierarchical clustering of Spearman correlation coefficients between peripheral blood and synovial fluid samples. (D) Differential expression analysis of global neutrophil marker expression between peripheral blood and synovial fluid. (E) Average expression of significantly differentially expressed markers per sample. (F) Comparison of geneand protein expression differences between blood and synovial fluid neutrophils identifies a hallmark synovial fluid phenotype. $\mathrm{HC}=$ healthy control; $\mathrm{IA}=$ inflammatory arthritis; SF = synovial fluid

\section{Figure 5. Continuous and discrete neutrophil phenotypes}

(A) UMAP embedding of single-cell CyTOF data separates blood neutrophils and synovial fluid cells. (B) Overclustering of neutrophils into 20 groups captures neutrophil heterogeneity across blood and synovial fluid. (C) Heterogeneity in marker expression between the 20 clusters. (D) Change in frequency of different neutrophil phenotypes across conditions. (E) Gradients of marker expression characterize synovial fluid neutrophils. Correlation between markers on a per-sample (F) and single-cell (G) level identifies clusters of co-expressed markers. $\mathrm{HC}=$ healthy control; $\mathrm{IA}=$ inflammatory arthritis; SF = synovial fluid

Figure 6. Progressive aging and response to interferon gamma recapitulate the synovial fluid phenotype in vitro

(A) Stimulation with IFNy extends the lifetime of neutrophils in vitro. (B) Effect of aging and IFNy on the expression of key surface markers. (C) Diffusion map of unstimulated and IFNy stimulated neutrophils cultured over 48 hours. (D) Expression of key surface markers on the diffusion map. 


\section{REFERENCES}

1. Nigrovic PA, Colbert RA, Holers VM, Ozen S, Ruperto N, Thompson SD, et al. Biological classification of childhood arthritis: roadmap to a molecular nomenclature. Nat Rev Rheumatol. 2021 May; 17(5):257-269.

2. Chang MH, Nigrovic PA. Antibody-dependent and -independent mechanisms of inflammatory arthritis. JCl Insight. 2019 Mar 7; 4(5).

3. Honig $M$, Peter HH, Jantscheff $P$, Grunert F. Synovial PMN show a coordinated up-regulation of CD66 molecules. J Leukoc Biol. 1999 Sep; 66(3):429-436.

4. Watson F, Robinson JJ, Phelan M, Bucknall RC, Edwards SW. Receptor expression in synovial fluid neutrophils from patients with rheumatoid arthritis. Ann Rheum Dis. 1993 May; 52(5):354-359.

5. Nurcombe HL, Bucknall RC, Edwards SW. Neutrophils isolated from the synovial fluid of patients with rheumatoid arthritis: priming and activation in vivo. Ann Rheum Dis. 1991 Mar; 50(3):147-153.

6. Quayle JA, Watson F, Bucknall RC, Edwards SW. Neutrophils from the synovial fluid of patients with rheumatoid arthritis express the high affinity immunoglobulin $G$ receptor, Fc gamma RI (CD64): role of immune complexes and cytokines in induction of receptor expression. Immunology. 1997 Jun; 91(2):266-273.

7. Emery P, Lopez AF, Burns GF, Vadas MA. Synovial fluid neutrophils of patients with rheumatoid arthritis have membrane antigen changes that reflect activation. Ann Rheum Dis. 1988 Jan; 47(1):34-39.

8. Miyabe Y, Miyabe C, Murooka TT, Kim EY, Newton GA, Kim ND, et al. Complement C5a Receptor is the Key Initiator of Neutrophil Adhesion Igniting Immune Complex-induced Arthritis. Sci Immunol. 2017 Jan; 2(7).

9. Romero V, Fert-Bober J, Nigrovic PA, Darrah E, Haque UJ, Lee DM, et al. Immune-mediated pore-forming pathways induce cellular hypercitrullination and generate citrullinated autoantigens in rheumatoid arthritis. Sci Transl Med. 2013 Oct 30; 5(209):209ra150.

10. Khandpur R, Carmona-Rivera C, Vivekanandan-Giri A, Gizinski A, Yalavarthi S, Knight JS, et al. NETs are a source of citrullinated autoantigens and stimulate inflammatory responses in rheumatoid arthritis. Sci Transl Med. 2013 Mar 27; 5(178):178ra140.

11. Chen M, Lam BK, Kanaoka Y, Nigrovic PA, Audoly LP, Austen KF, et al. Neutrophil-derived leukotriene B4 is required for inflammatory arthritis. J Exp Med. 2006 Apr 17; 203(4):837842.

12. Chatham WW, Swaim R, Frohsin H, Jr., Heck LW, Miller EJ, Blackburn WD, Jr. Degradation of human articular cartilage by neutrophils in synovial fluid. Arthritis Rheum. 1993 Jan; 36(1):51-58.

13. Elliott ER, Van Ziffle JA, Scapini P, Sullivan BM, Locksley RM, Lowell CA. Deletion of Syk in neutrophils prevents immune complex arthritis. J Immunol. 2011 Oct 15; 187(8):4319-4330.

14. Wipke BT, Allen PM. Essential role of neutrophils in the initiation and progression of a murine model of rheumatoid arthritis. J Immunol. 2001 Aug 1; 167(3):1601-1608.

15. Monach PA, Nigrovic PA, Chen M, Hock H, Lee DM, Benoist C, et al. Neutrophils in a mouse model of autoantibody-mediated arthritis: critical producers of Fc receptor gamma, the receptor for $\mathrm{C5a}$, and lymphocyte function-associated antigen 1. Arthritis Rheum. 2010 Mar; 62(3):753-764.

16. Wang JX, Bair AM, King SL, Shnayder R, Huang YF, Shieh CC, et al. Ly6G ligation blocks recruitment of neutrophils via a beta2-integrin-dependent mechanism. Blood. 2012 Aug 16; 120(7):1489-1498.

17. Cunin P, Lee PY, Kim E, Schmider AB, Cloutier N, Pare A, et al. Differential attenuation of beta2 integrin-dependent and -independent neutrophil migration by Ly6G ligation. Blood Adv. 2019 Feb 12; 3(3):256-267. 
18. Grieshaber-Bouyer R, Nigrovic PA. Neutrophil Heterogeneity as Therapeutic Opportunity in Immune-Mediated Disease. Front Immunol. 2019; 10:346.

19. Grieshaber-Bouyer R, Radtke FA, Cunin P, Stifano G, Levescot A, Vijaykumar B, et al. The neutrotime transcriptional signature defines a single continuum of neutrophils across biological compartments. Nat Commun. 2021 May 17; 12(1):2856.

20. Adrover JM, Nicolas-Avila JA, Hidalgo A. Aging: A Temporal Dimension for Neutrophils. Trends Immunol. 2016 May; 37(5):334-345.

21. Zhang D, Chen G, Manwani D, Mortha A, Xu C, Faith JJ, et al. Neutrophil ageing is regulated by the microbiome. Nature. 2015 Sep 24; 525(7570):528-532.

22. Bai M, Grieshaber-Bouyer R, Wang J, Schmider AB, Wilson ZS, Zeng L, et al. CD177 modulates human neutrophil migration through activation-mediated integrin and chemoreceptor regulation. Blood. 2017 Nov 9; 130(19):2092-2100.

23. Bandura DR, Baranov VI, Ornatsky OI, Antonov A, Kinach R, Lou X, et al. Mass cytometry: technique for real time single cell multitarget immunoassay based on inductively coupled plasma time-of-flight mass spectrometry. Anal Chem. 2009 Aug 15; 81(16):6813-6822.

24. Lee PY, Wang JX, Parisini E, Dascher CC, Nigrovic PA. Ly6 family proteins in neutrophil biology. J Leukoc Biol. 2013 Oct; 94(4):585-594.

25. Ericson JA, Duffau P, Yasuda K, Ortiz-Lopez A, Rothamel K, Rifkin IR, et al. Gene expression during the generation and activation of mouse neutrophils: implication of novel functional and regulatory pathways. PLoS One. 2014; 9(10):e108553.

26. Howe KL, Achuthan P, Allen J, Allen J, Alvarez-Jarreta J, Amode MR, et al. Ensembl 2021. Nucleic Acids Res. 2021 Jan 8; 49(D1):D884-D891.

27. Isles HM, Herman KD, Robertson AL, Loynes CA, Prince LR, Elks PM, et al. The CXCL12/CXCR4 Signaling Axis Retains Neutrophils at Inflammatory Sites in Zebrafish. Front Immunol. 2019; 10:1784.

28. Goulding NJ, Knight SM, Godolphin JL, Guyre PM. Increase in neutrophil Fc gamma receptor I expression following interferon gamma treatment in rheumatoid arthritis. Ann Rheum Dis. 1992 Apr; 51(4):465-468.

29. Akerley WL, 3rd, Guyre PM, Davis BH. Neutrophil activation through high-affinity Fc gamma receptor using a monomeric antibody with unique properties. Blood. 1991 Feb 1; 77(3):607615.

30. Reinisch W, Tillinger W, Lichtenberger C, Gangl A, Willheim M, Scheiner O, et al. In vivo induction of HLA-DR on human neutrophils in patients treated with interferon-gamma. Blood. 1996 Apr 1; 87(7):3068.

31. Gosselin EJ, Wardwell K, Rigby WF, Guyre PM. Induction of MHC class II on human polymorphonuclear neutrophils by granulocyte/macrophage colony-stimulating factor, IFNgamma, and IL-3. J Immunol. 1993 Aug 1; 151(3):1482-1490.

32. Langereis JD, Pickkers P, de Kleijn S, Gerretsen J, de Jonge MI, Kox M. Spleen-derived IFNgamma induces generation of PD-L1(+)-suppressive neutrophils during endotoxemia. J Leukoc Biol. 2017 Dec; 102(6):1401-1409.

33. Wang JF, Wang YP, Xie J, Zhao ZZ, Gupta S, Guo Y, et al. Upregulated PD-L1 delays human neutrophil apoptosis and promotes lung injury in an experimental mouse model of sepsis. Blood. 2021 Sep 2; 138(9):806-810.

34. de Kleijn S, Langereis JD, Leentjens J, Kox M, Netea MG, Koenderman L, et al. IFN-gammastimulated neutrophils suppress lymphocyte proliferation through expression of PD-L1. PLoS One. 2013; 8(8):e72249.

35. Massena S, Christoffersson G, Vagesjo E, Seignez C, Gustafsson K, Binet F, et al. Identification and characterization of VEGF-A-responsive neutrophils expressing CD49d, VEGFR1, and CXCR4 in mice and humans. Blood. 2015 Oct 22; 126(17):2016-2026.

36. Ropes MW, Bauer W. Synovial fluid changes in joint disease. Cambridge, Mass.,: Harvard Univ. Press, 1953. 
37. Xie X, Shi Q, Wu P, Zhang X, Kambara H, Su J, et al. Single-cell transcriptome profiling reveals neutrophil heterogeneity in homeostasis and infection. Nat Immunol. 2020 Sep; 21(9):1119-1133.

38. Ballesteros I, Rubio-Ponce A, Genua M, Lusito E, Kwok I, Fernandez-Calvo G, et al. Cooption of Neutrophil Fates by Tissue Environments. Cell. 2020 Nov 25; 183(5):1282-1297 e1218.

39. Brach MA, deVos S, Gruss HJ, Herrmann F. Prolongation of survival of human polymorphonuclear neutrophils by granulocyte-macrophage colony-stimulating factor is caused by inhibition of programmed cell death. Blood. 1992 Dec 1; 80(11):2920-2924.

40. Stevens CR, Williams RB, Farrell AJ, Blake DR. Hypoxia and inflammatory synovitis: observations and speculation. Ann Rheum Dis. 1991 Feb; 50(2):124-132.

41. Klimiuk PA, Yang $\mathrm{H}$, Goronzy JJ, Weyand CM. Production of cytokines and metalloproteinases in rheumatoid synovitis is T cell dependent. Clin Immunol. 1999 Jan; 90(1):65-78.

42. Berner B, Akca D, Jung T, Muller GA, Reuss-Borst MA. Analysis of Th1 and Th2 cytokines expressing CD4+ and CD8+ T cells in rheumatoid arthritis by flow cytometry. J Rheumatol. 2000 May; 27(5):1128-1135.

43. Kang YM, Zhang X, Wagner UG, Yang H, Beckenbaugh RD, Kurtin PJ, et al. CD8 T cells are required for the formation of ectopic germinal centers in rheumatoid synovitis. J Exp Med. 2002 May 20; 195(10):1325-1336.

44. Wu HJ, Sawaya H, Binstadt B, Brickelmaier M, Blasius A, Gorelik L, et al. Inflammatory arthritis can be reined in by CpG-induced DC-NK cell cross talk. J Exp Med. 2007 Aug 6; 204(8):1911-1922.

45. Kim HY, Kim HJ, Min HS, Kim S, Park WS, Park SH, et al. NKT cells promote antibodyinduced joint inflammation by suppressing transforming growth factor beta1 production. J Exp Med. 2005 Jan 3; 201(1):41-47.

46. Zhao S, Grieshaber-Bouyer R, Rao DA, Kolb P, Chen H, Andreeva I, et al. JAK inhibition prevents the induction of pro-inflammatory HLA-DR(+) CD90(+) RA synovial fibroblasts by IFN. Arthritis Rheumatol. 2021 Aug 25.

47. Nabbe KC, van Lent PL, Holthuysen AE, Kolls JK, Verbeek S, van den Berg WB. FcgammaRI up-regulation induced by local adenoviral-mediated interferon-gamma production aggravates chondrocyte death during immune complex-mediated arthritis. Am J Pathol. 2003 Aug; 163(2):743-752.

48. Manoury-Schwartz B, Chiocchia G, Bessis N, Abehsira-Amar O, Batteux F, Muller S, et al. High susceptibility to collagen-induced arthritis in mice lacking IFN-gamma receptors. J Immunol. 1997 Jun 1; 158(11):5501-5506.

49. Vermeire K, Heremans H, Vandeputte M, Huang S, Billiau A, Matthys P. Accelerated collagen-induced arthritis in IFN-gamma receptor-deficient mice. J Immunol. 1997 Jun 1; 158(11):5507-5513.

50. Cannon GW, Pincus SH, Emkey RD, Denes A, Cohen SA, Wolfe F, et al. Double-blind trial of recombinant gamma-interferon versus placebo in the treatment of rheumatoid arthritis. 1989. Arthritis Rheum. 2008 Feb; 58(2 Suppl):S79-88.

51. Veys EM, Mielants H, Verbruggen G, Grosclaude JP, Meyer W, Galazka A, et al. Interferon gamma in rheumatoid arthritis--a double blind study comparing human recombinant interferon gamma with placebo. J Rheumatol. 1988 Apr; 15(4):570-574.

52. Marini O, Costa S, Bevilacqua D, Calzetti F, Tamassia N, Spina C, et al. Mature CD10(+) and immature CD10(-) neutrophils present in G-CSF-treated donors display opposite effects on T cells. Blood. 2017 Mar 9; 129(10):1343-1356. 

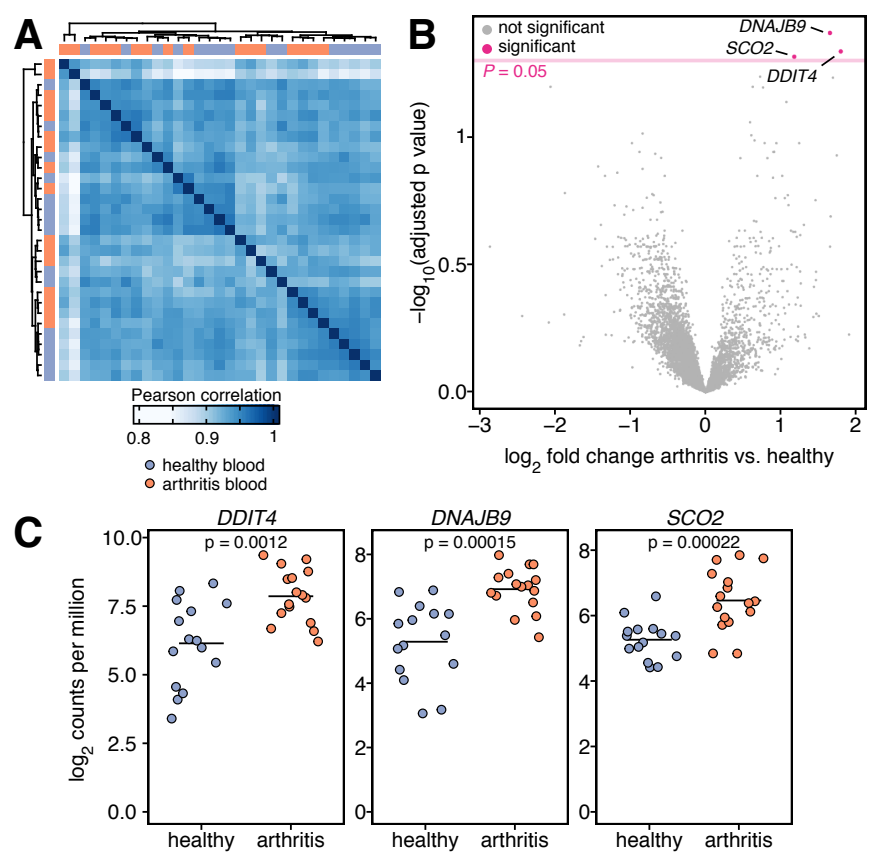

Figure 1. Transcriptomic similarity of blood neutrophils from healthy controls and patients with inflammatory arthritis (A) Hierarchical clustering of Pearson correlation coefficients between individual blood samples based on the expression of all genes reveals complete overlap between the two groups. (B) Volcano plot of differentially expressed genes (at FDR 0.05) between blood neutrophils from healthy and arthritic donors. (C) DNAJB9, DDIT4 and SCO2 are overexpressed in blood of inflammatory arthritis patients compared to healthy controls. $\mathrm{N}=15$ healthy controls, $\mathrm{N}=16$ arthritis patients. 

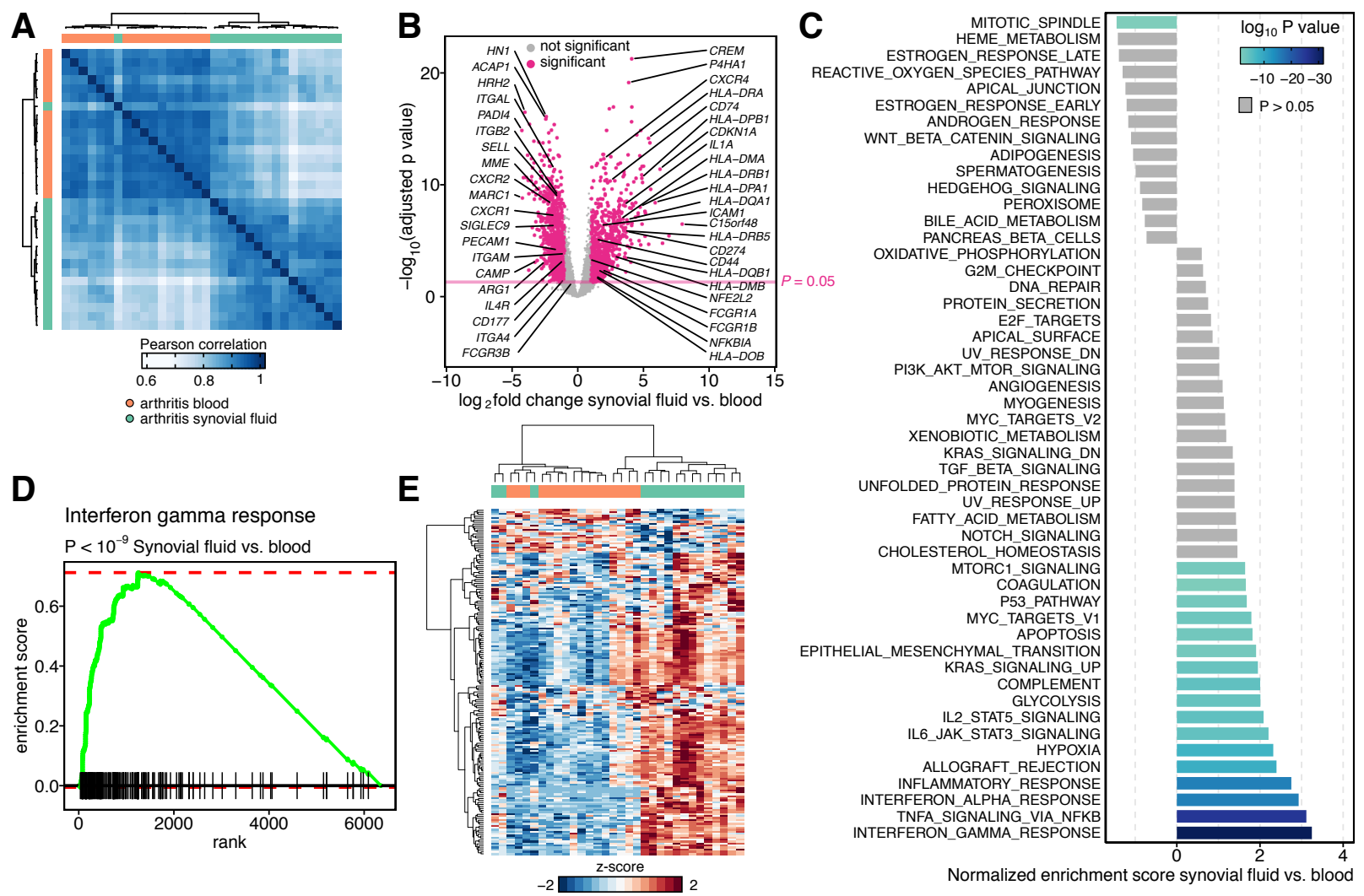

Normalized enrichment score synovial fluid vs. blood

Figure 2. Synovial fluid neutrophils are enriched for IFNy response genes

(A) Hierarchical clustering of Pearson correlation coefficients between paired peripheral blood and synovial fluid samples based on the expression of all genes shows strong separation based on tissue. (B) 1,657/6,350 genes are differentially expressed at $\log _{2}$ fold change $\geq 1$ and FDR of 0.05 between peripheral blood and synovial fluid neutrophils. (C) Gene Set Enrichment Analysis of differentially expressed genes in synovial fluid vs. blood neutrophils. (D) Enrichment plot of the IFNy response signature in synovial fluid neutrophils. (E) Expression heatmap of IFNy response genes in synovial fluid neutrophils reveals strong separation between blood and synovial fluid. $\mathrm{N}=16$ paired blood and synovial fluid inflammatory arthritis samples. 
bioRxiv preprint doi: https://doi.org/10.1101/2021.11.16.465905; this version posted November 18, 2021. The copyright holder for this preprint (which was not certified by peer review) is the author/funder. All rights reserved. No reuse allowed without permission.

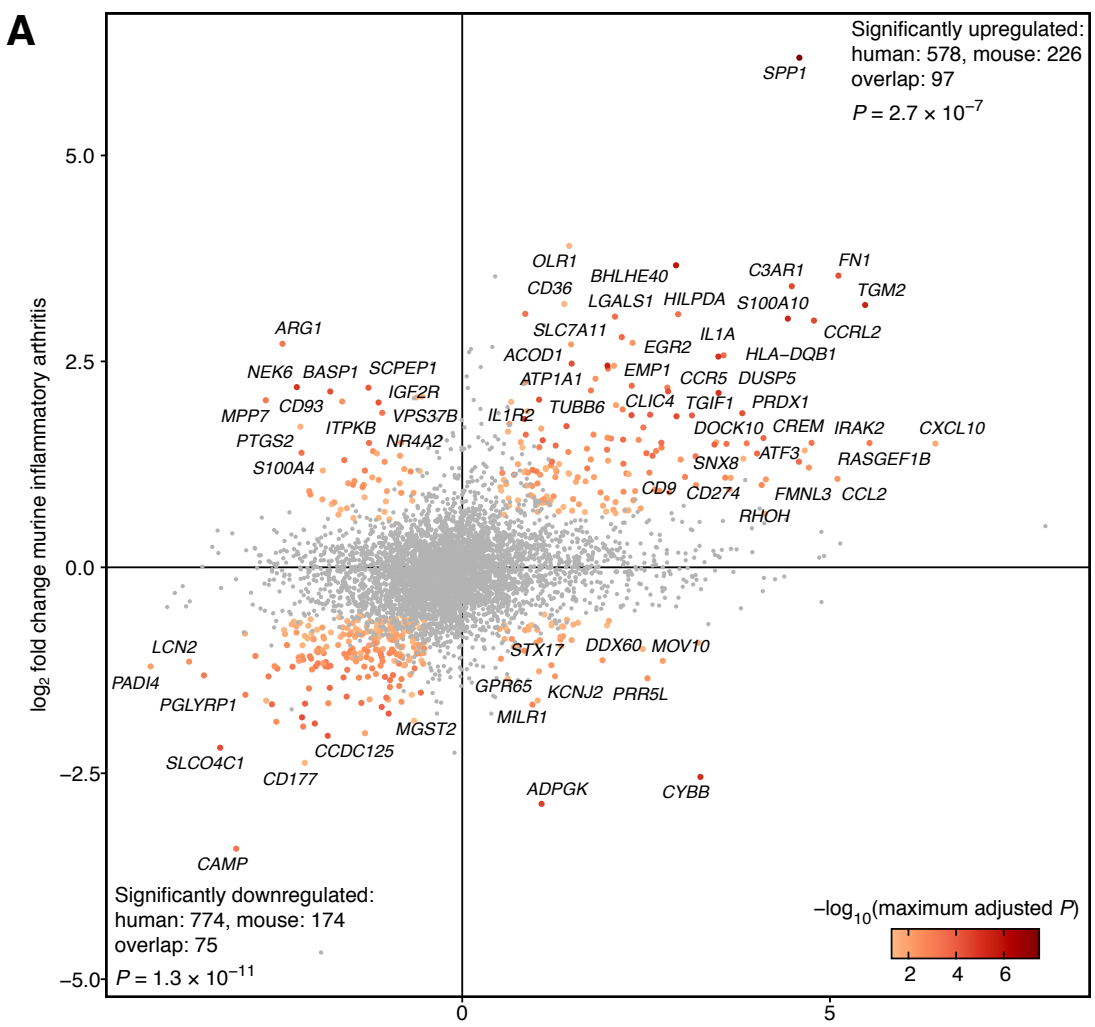

$\log _{2}$ fold change human inflammatory arthritis

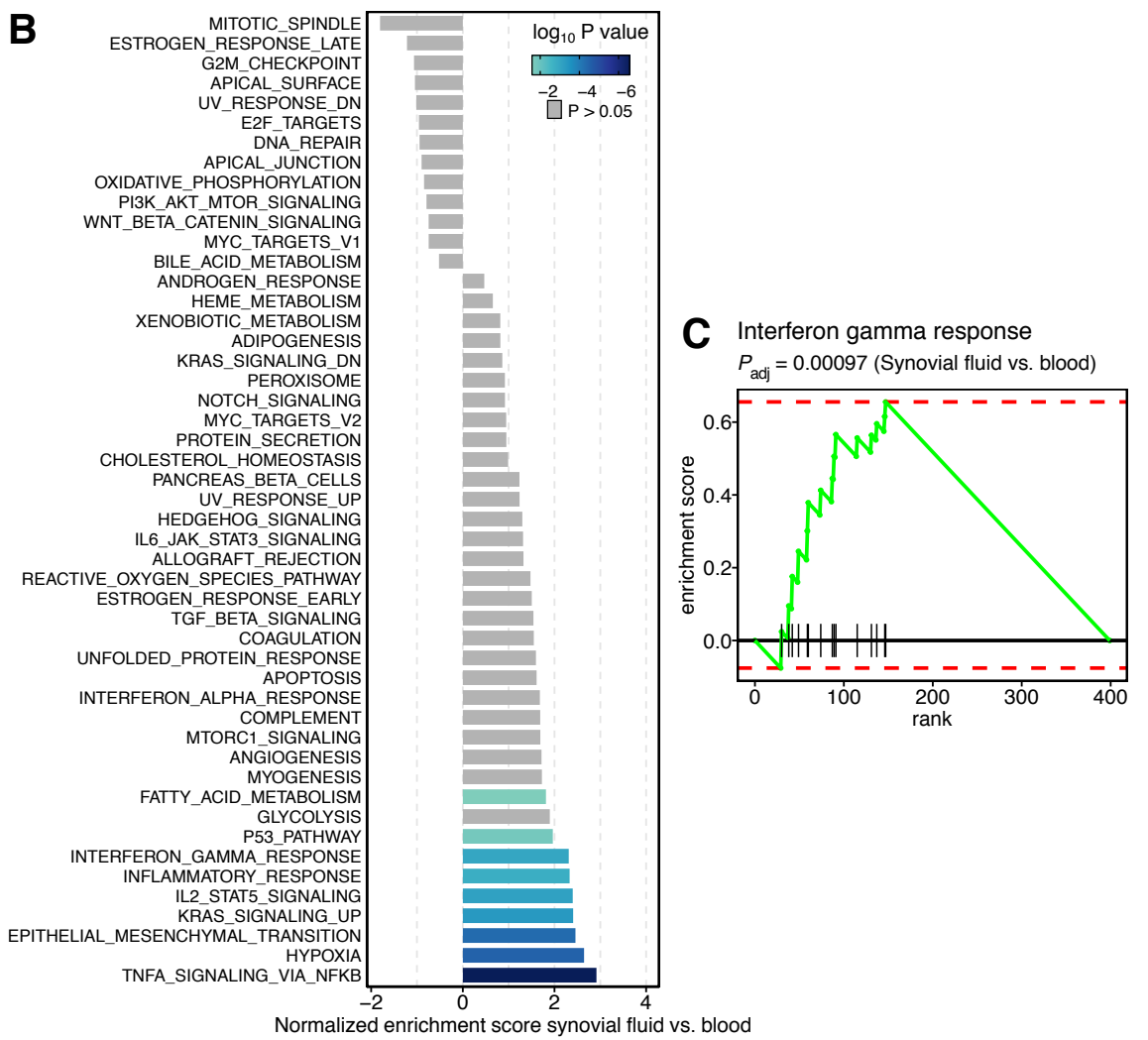

Figure 3. Cross species analysis of neutrophil gene expression in inflamed synovial fluid

(A) Depicted is the $\log _{2}$ fold change of gene expression in human ( $x$ ) vs. murine (y) synovial fluid neutrophils compared to blood neutrophils. Only genes with with one-to-one orthologs are shown and genes with adjusted $\mathrm{P}<0.05$ in both comparisons and I log2 fold change I $\geq 0.75$ are highlighted. Genes are conservatively colored by highest $P$ value. (B) Significantly differentially expressed genes were ranked by $\log _{2}$ fold change and Gene Set Enrichment Analysis was performed on hallmark gene sets. (C) Enrichment plot of the hallmark gene set "Interferon gamma response". Only genes with one-to-one orthologs between mice and humans are shown; for gene symbols, the human symbol is shown. 
A

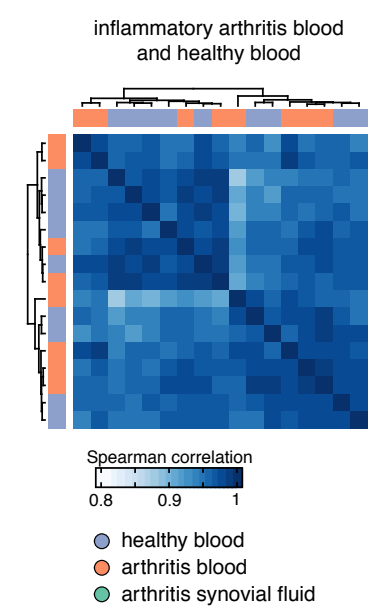

B

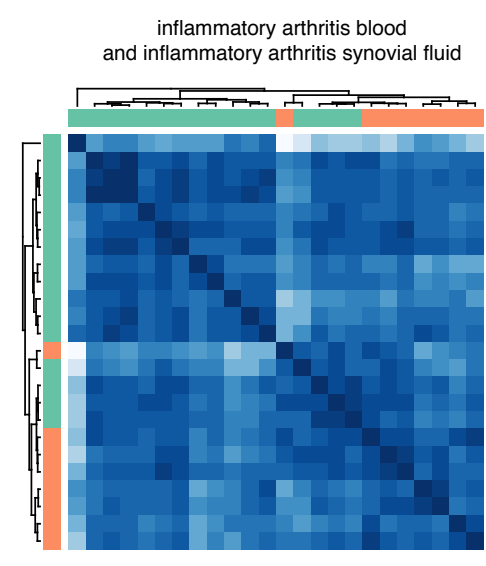

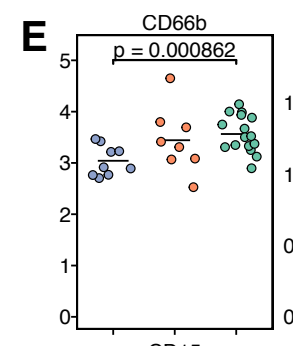
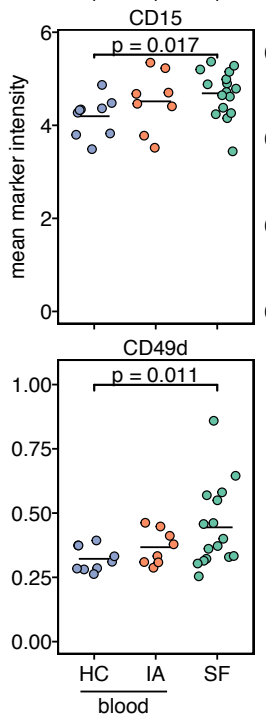
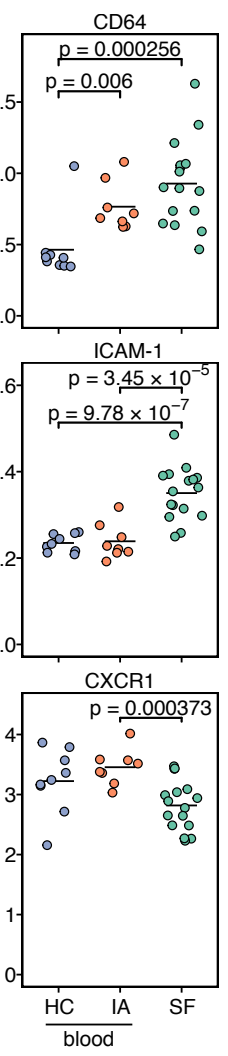
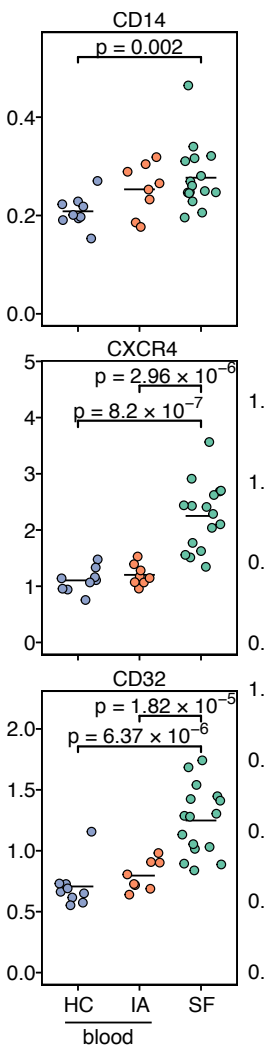

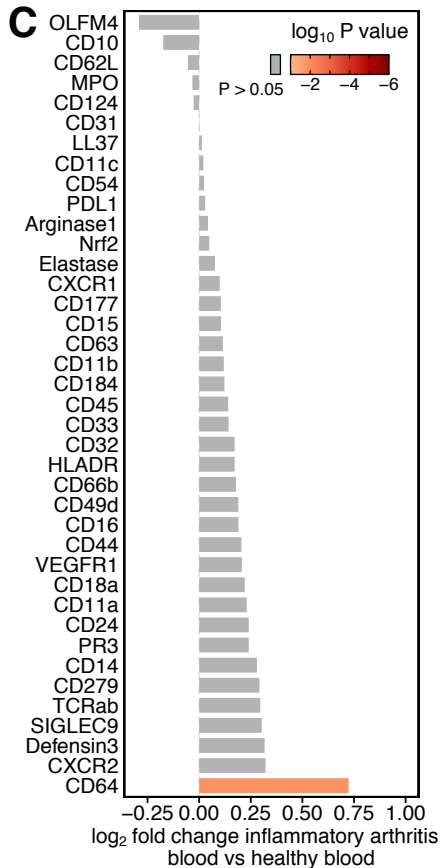
blood vs healthy blood

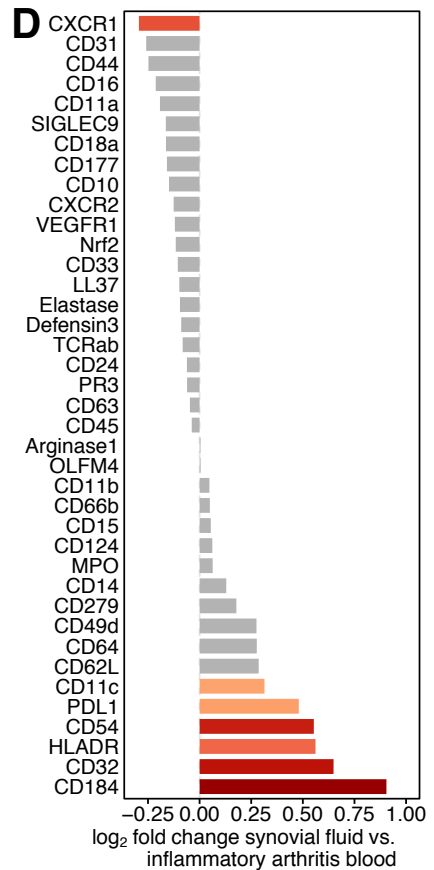

$\mathbf{F}$
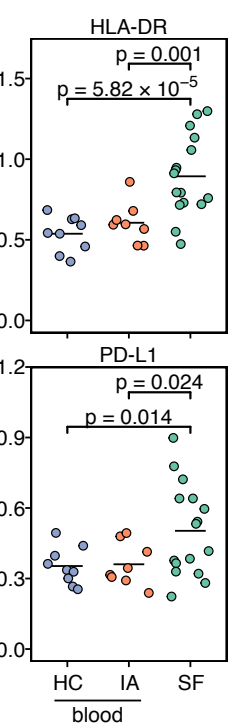

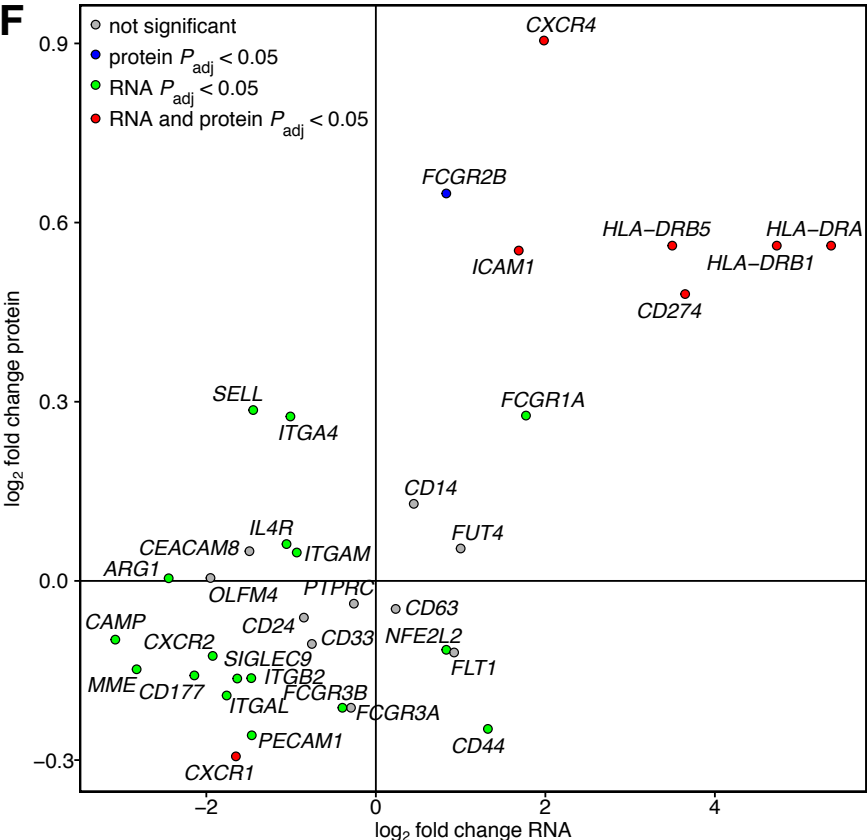

Figure 4. Mass cytometry analysis of neutrophils

(A) Hierarchical clustering of Spearman correlation coefficients between blood neutrophils from healthy donors and patients with inflammatory arthritis based on global neutrophil protein expression. (B) Differential expression analysis of global neutrophil marker expression in the peripheral blood. (C) Hierarchical clustering of Spearman correlation coefficients between peripheral blood and synovial fluid samples. (D) Differential expression analysis of global neutrophil marker expression between peripheral blood and synovial fluid. (E) Average expression of significantly differentially expressed markers per sample. (F) Comparison of gene- and protein expression differences between blood and synovial fluid neutrophils identifies a hallmark synovial fluid phenotype.

$\mathrm{HC}=$ healthy control; IA = inflammatory arthritis; SF = synovial fluid

\section{Figure 5. Continuous and discrete neutrophil phenotypes}

(A) UMAP embedding of single-cell CyTOF data separates blood neutrophils and synovial fluid cells. (B) Overclustering of neutrophils into 20 groups captures neutrophil heterogeneity across blood and synovial fluid. (C) Heterogeneity in marker expression between the 20 clusters. (D) Change in frequency of different neutrophil phenotypes across conditions. (E) Gradients of marker expression characterize synovial fluid neutrophils. Correlation between markers on a per-sample (F) and single-cell (G) level identifies clusters of co-expressed markers. $\mathrm{HC}=$ healthy control; IA = inflammatory arthritis; SF = synovial fluid 
Figure 5 (Legend on previous page)
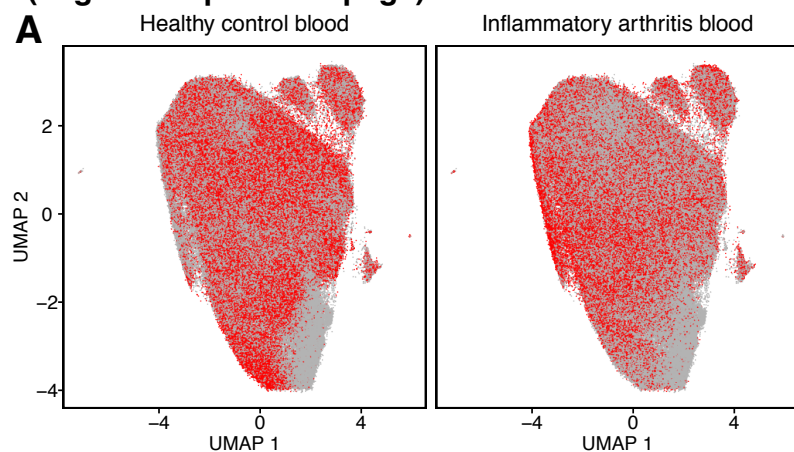

C

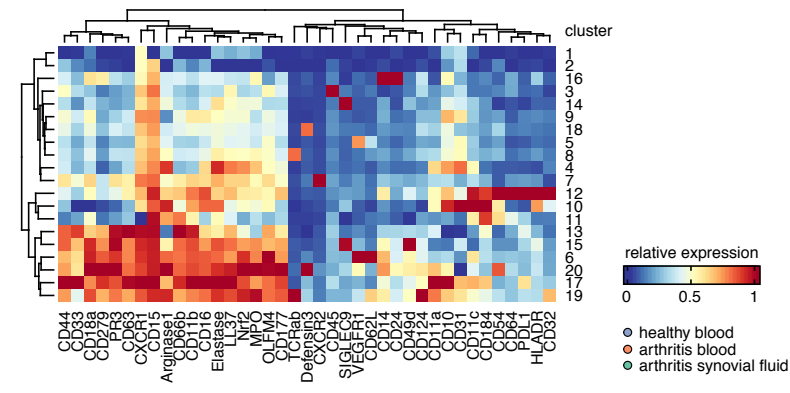

D

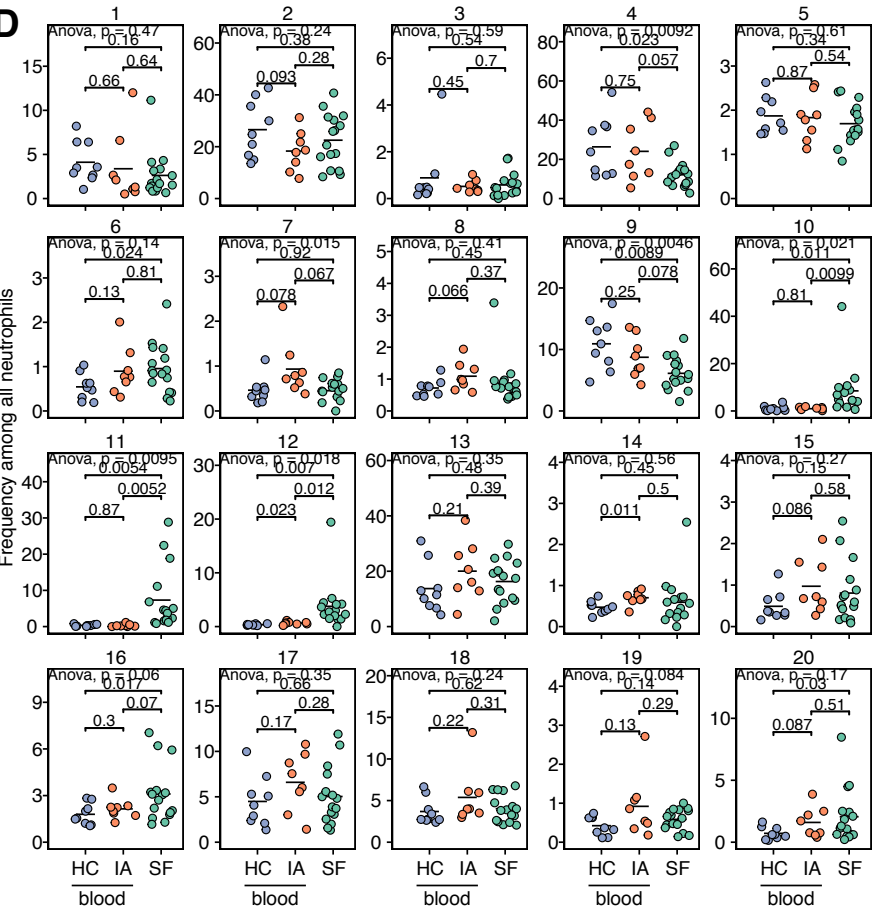

$\mathbf{F}$

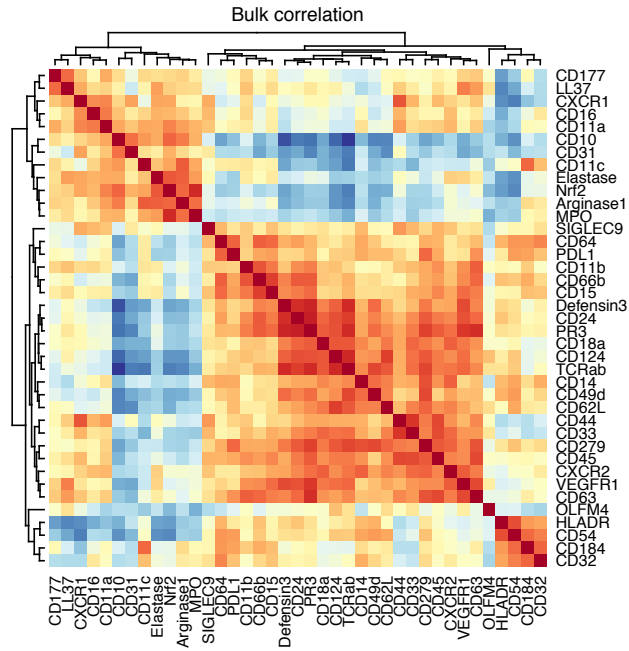

Synovial fluid
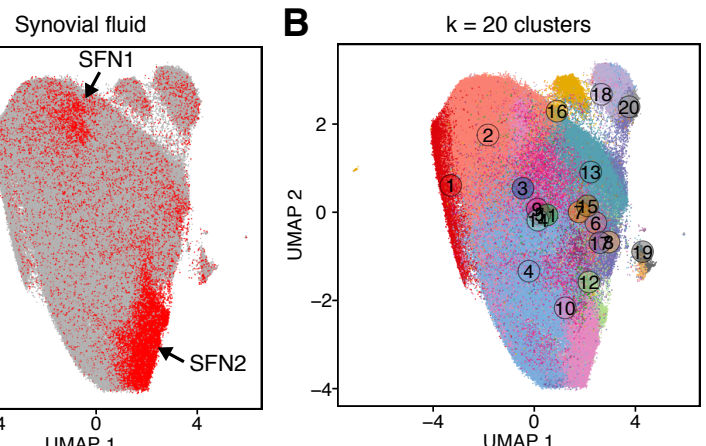

E

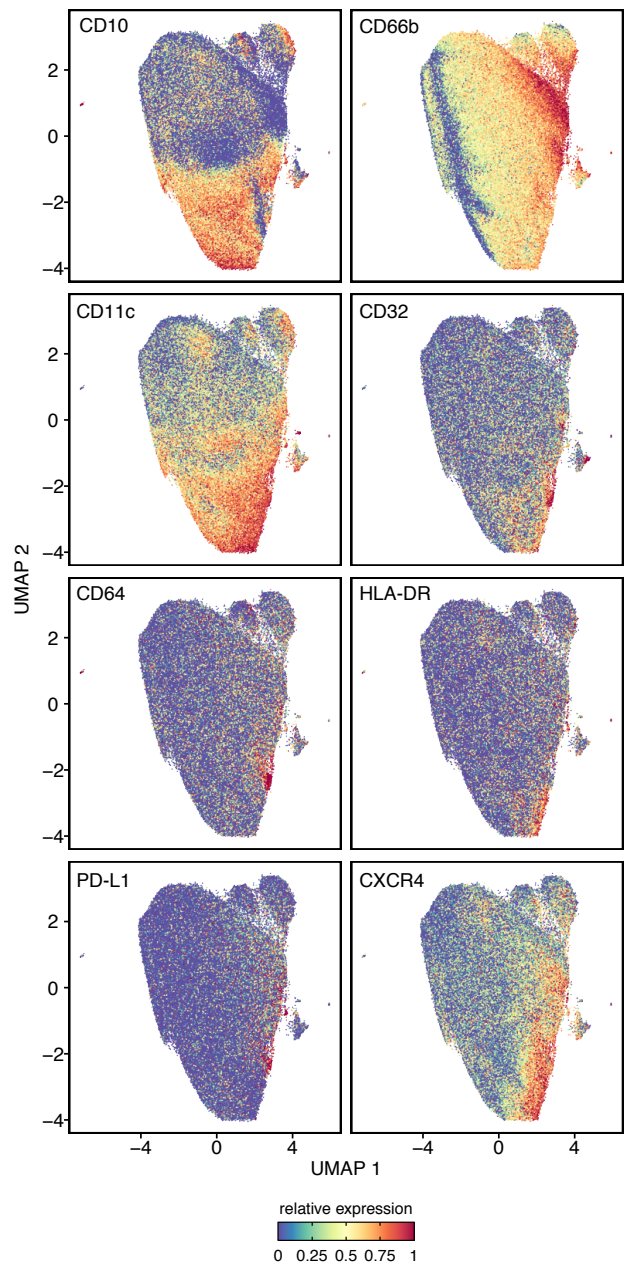

G

spearman correlation

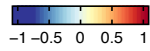

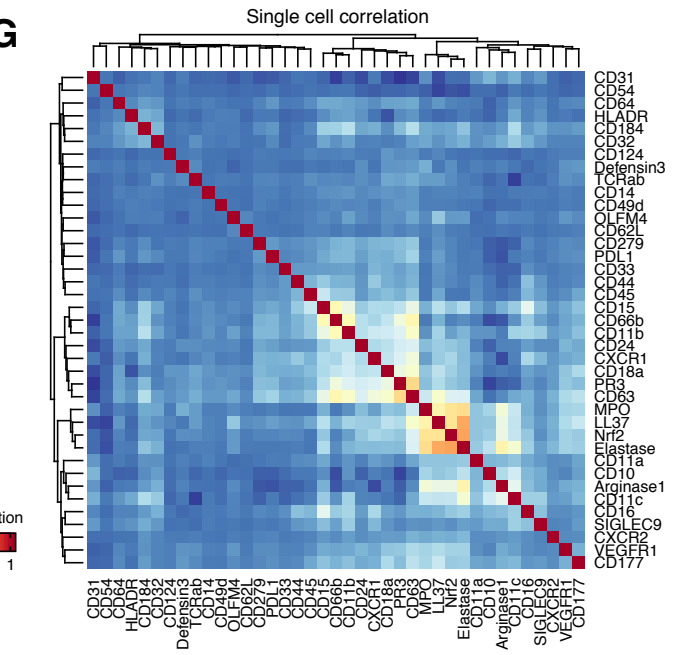


bioRxiv preprint doi: https://doi.org/10.1101/2021.11.16.465905; this version posted November 18, 2021. The copyright holder for this preprint (which was not certified by peer review) is the author/funder. All rights reserved. No reuse allowed without permission.

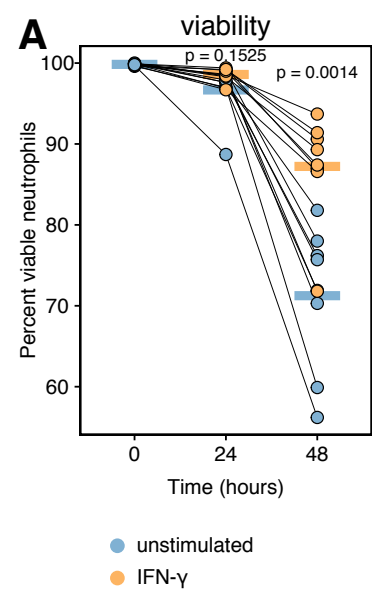

B

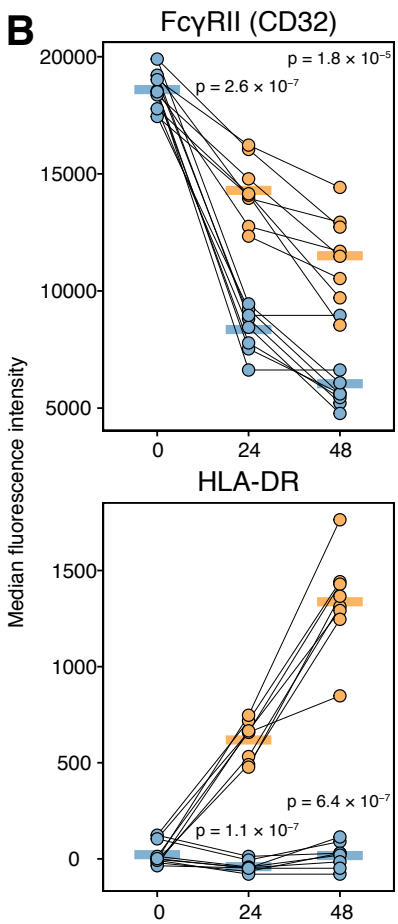

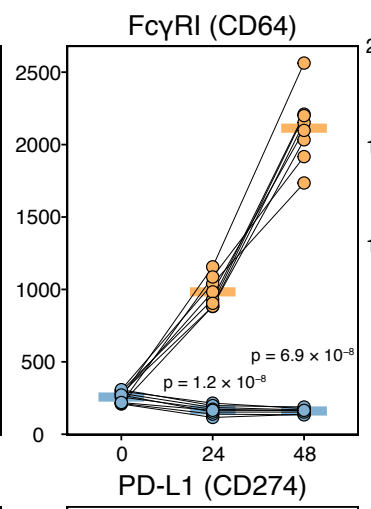
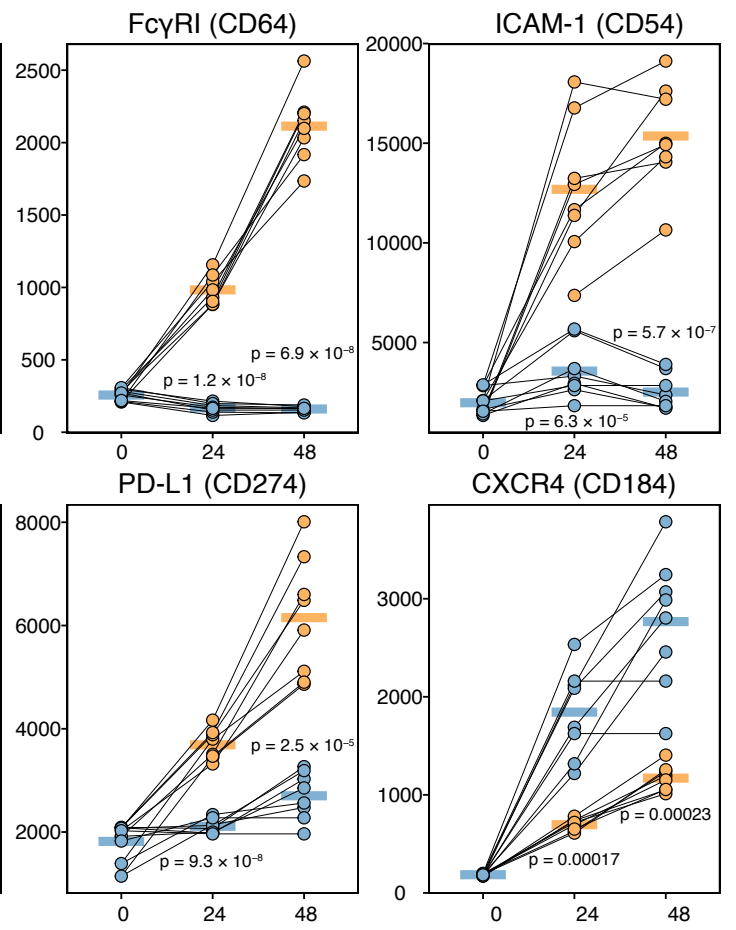

CXCR4 (CD184)

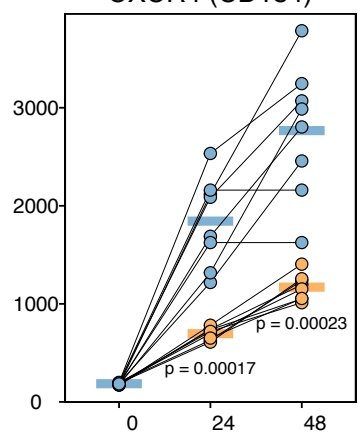

C
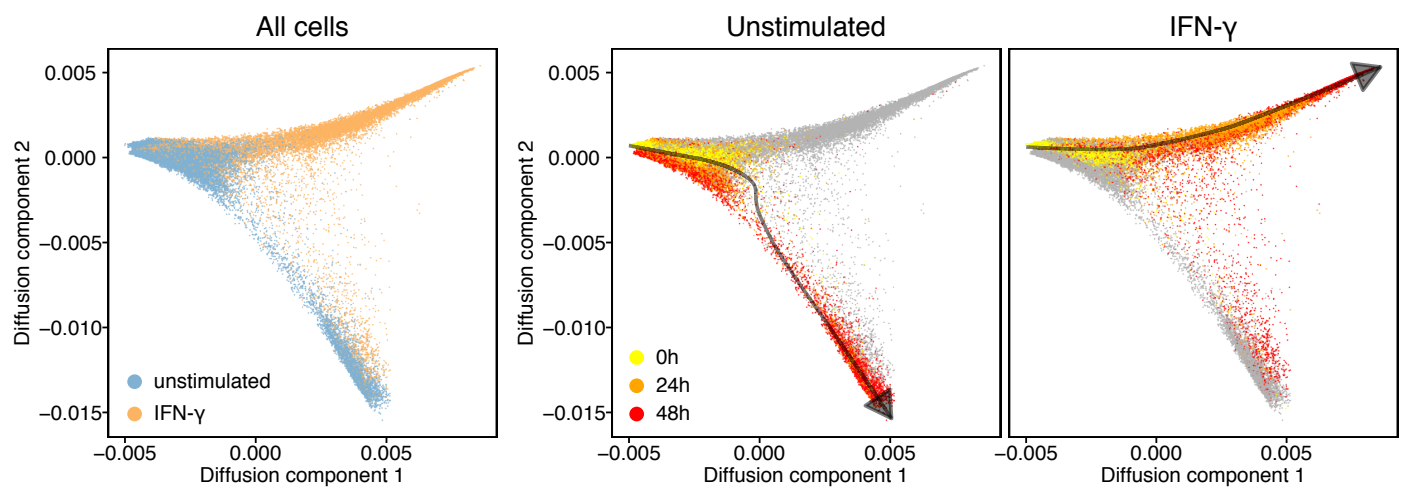

D
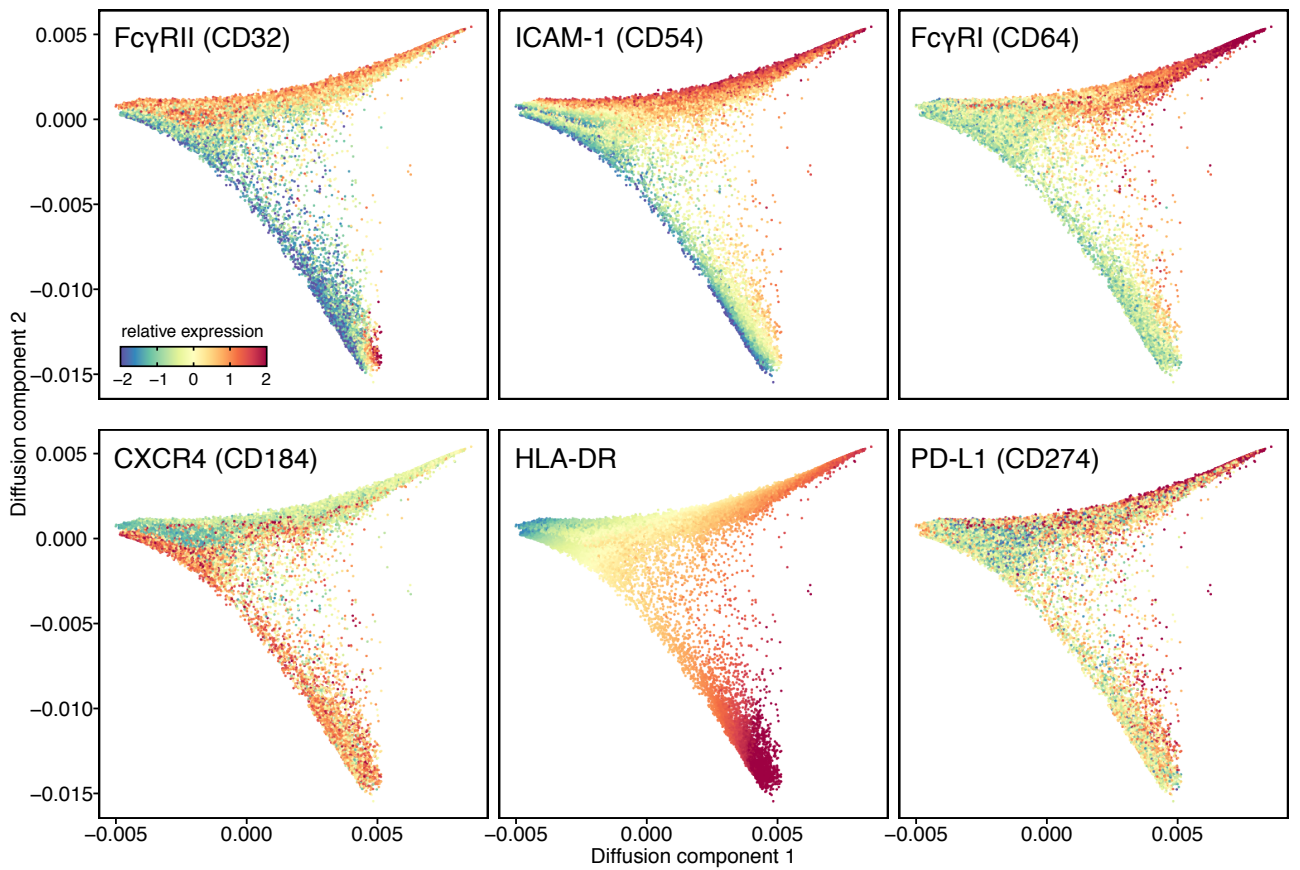

Figure 6. Progressive aging and response to interferon gamma recapitulate the synovial fluid phenotype in vitro (A) Stimulation with IFNY extends the lifetime of neutrophils in vitro. (B) Effect of aging and IFNY on the expression of key surface markers. (C) Diffusion map of unstimulated and IFNY stimulated neutrophils cultured over 48 hours. (D) Expression of key surface markers on the diffusion map. 